\section{Nutzen und Risiken der Rotavirus-Impfung}

\begin{abstract}
Jüngste Studien aus den USA hatten belegt, dass das Risiko für Invaginationen in der ersten Woche nach einer Impfung gegen Rotaviren leicht erhöht ist. Nun stellt sich die Frage, ob man die Impfung weiterhin durchführen soll. Ja, sagen die Autoren einer aktuellen Studie aus der Zeitschrift Pediatrics.
\end{abstract}

D Impfung gegen Rotaviren ist in die Diskussion gekommen, nachen gezeigt hatte, dass die Invaginationsrate nach der ersten Impfung erhöht ist. In Frankreich war es zu einem Todesfall gekommen. Aus diesem Grund verdient die vorliegende Untersuchung aus dem Center for Disease Control in Atlanta besondere Beachtung. Hier wurden die Invaginationsraten bei jungen Säuglingen in den Jahren von 2000-2005 vor Einführung der Rotavirusimpfung mit den Jahren von 2007-2013 nach Einführung der Impfung verglichen.

Dabei wurde festgestellt, dass die Invaginationshäufigkeit nur bei Kindern in der Altersgruppe von 8-11 Wochen um etwa $46-101 \%$ erhöht war, nicht jedoch bei älteren Kindern. Das Risiko eidem sich in den Nachzulassungsstudi- ner Invagination war nur in der ersten Woche nach der ersten Impfung erhöht. In den Jahren 2011 und 2013 war der Effekt überhaupt nicht nachweisbar. Insgesamt war das Risiko für Invaginationen in der Altersgruppe von 8-11 Wochen dermaßen gering, dass eine Erhöhung der Invaginationshäufigkeit $a b$ dem Jahr 2007 im gesamten ersten Lebensjahr überhaupt nicht nachweisbar war. Der Verzicht auf die Impfung schaltet das Invaginationsrisiko übrigens keineswegs aus. Es beträgt immer noch 11,7 pro 100.000 Kinder.

Die Autoren um Jacqueline E. Tate sind der Überzeugung, dass der starke Rückgang von Rotaviruserkrankungen durch die Impfung gegen Rotaviren massiv gegenüber dem gering erhöhten Invaginationsrisiko überwiegt. Die
Wissenschaftler rechnen vor, dass im Zeitraum von 2007-2011 in den USA mehr als 176.000 Krankenhausaufnahmen wegen Rotavirusinfektionen verhindert worden waren sowie 242.000 Besuche einer Notfallambulanz und 1,1 Millionen Praxisbesuche. Aus diesem Grund ist nach Meinung der Autoren der Nutzen der Impfung weit größer als die geringe Erhöhung des Invaginationsrisikos.

Tate JE et al. Intussusception rates before and after the introduction of rotavirus vaccine. Pediatrics 2016;138: pii: e20161082

\section{Kommentar}

Leider sind viele Kinderärzte in Deutschland dazu übergegangen, Eltern von jungen Säuglingen von der Impfung gegen Rotaviren abzuraten. Dies scheint kein guter Ratschlag zu sein. In einer Zeit, in der Kleinkinder Kindertagesstätten besuchen und beide Eltern berufstätig sind, sollten Kinderärzte ergebnisoffen und nicht mit einem paternalistischen Vorurteil behaftet beraten.

Dr. Hartmut Koch

\section{Gefäßmalformation im Knie - was tun?}

\section{Eine synoviale Gefäßmalformation im Bereich der Kniestrukturen ist etwas sehr Seltenes. Aufgrund unspezifischer klinischer Zeichen wie Schmerzen, Schwellung und Bewegungseinschränkung wird die Diagnose oftmals verzögert gestellt. Im aktuellen Journal of Pediatrics wird zu dieser Thematik eine interessante Beobachtung einer Arbeitsgruppe aus Padua mitgeteilt.}

$B^{c}$ ei einem 7 Jahre alten Mädchen wurde aufgrund intermittierender Schmerzen des rechten Knies in der Annahme einer juvenilen idiopathischen Oligoarthritis eine Kortisontherapie durchgeführt. Klinisch bestand eine Schwellung korrespondierend zur Region des Hoffa-Fettkörpers bei korrekter Knieausrichtung und ohne Laxheit der Gelenkbänder. Aufgrund fehlender Besserung der Symptome wurde die Patientin einer eingehenden bildgebenden Diagnostik unterzogen. In der Kernspintomografie zeigte sich nun überraschend eine ungewöhnliche Gefäßstruktur der Kniesynovia. Die Methode de- tektierte ursächlich ein Hämangiom beziehungsweise eine venöse Malformation (Abb. 1). In der dynamischen Farbdopplersonografie bot sich das Bild einer synovialen Gefäßmalformation mit umschriebener Interruption der medialen Patellarsehne (Abb. 2).

Beltrame $V$ et al. Synovial hemangioma of infrapatellar (Hoffa) fat pad: Magnetic resonance imaging and ultrasound features. J Pediatr 2016;172:222-3

\section{Kommentar}

Symptomatik, Diagnostik und Klassifikation der juvenilen idiopathischen Arthritis sollen

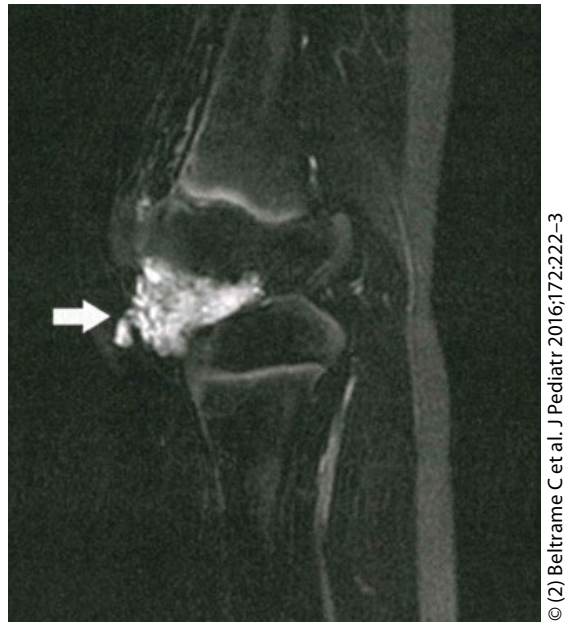

Abb. 1: Synoviale Gefäß- oder venöse "low-flow"-Malformation im saggitalen T2gewichteten Kernspintomogramm

an dieser Stelle nicht diskutiert werden. In jedem Fall ist die Kortisontherapie bei diesem Mädchen unverständlich. Der infrapatellare Fettkörper (Corpus adiposum infrapatellare; Synonym: Hoffa-Fettkörper) ist eine intrakapsuläre extrasynoviale Struktur, 